\title{
An Extended Fuzzy Logic Method for Watershed
}

\author{
Ling Zhang ${ }^{1}$, Ming Zhang ${ }^{2}$, H.D. Cheng ${ }^{2}$ \\ ${ }^{1}$ School of Mathematics and System Sciences, Shandong University, Jinan, Shandong \\ 250100 \\ ${ }^{2}$ Department of Computer Science, Utah State University, Logan, UT 84322
}

\begin{abstract}
Fuzzy Logic was intruded by Lotfi A. Zadeh in 1965. It is for solving uncertainty and ambiguity problems. Fuzzy logic is a multivalued logic defined in $[0,1]$. However, there are a lot of paradoxes, as proposition, can not be described in Fuzzy Logic. In this paper, we will define an extended fuzzy logic and applied it on watershed segmentation method in image processing.
\end{abstract}

Keywords: Extended Fuzzy Logic, Segmentation, Watershed, Image Processing

\section{Introduction}

Fuzzy logic[1] is a successful method in modeling vagueness and uncertainty problems for about 40 years. It is widely used in engineering. However, fuzzy logic can only modeling part of vagueness problems. There are more problems which cannot be solved by fuzzy logic. For example, the weather forecast suggests that there is $40 \%$ chance of rain tomorrow according past year's weather and $50 \%$ chance of no rain according today's weather. There are a lot of factors may affect tomorrow's weather, such as altitude, humidity, etc. We only know part of them and there are some conditions that we don't know or indeterminate. The more we know, the more we don't know. In fuzzy Logic, it describes the degree of uncertain for the known conditions or factors. But the world is full of indeterminacy.

In this paper, an extended fuzzy logic method will be defined. A new parameter $I$ is introduced into fuzzy logic, which stands for indeterminate factors. This extension is closer to the human mind reasoning and it is more powerful. Then the extended fuzzy logic method is applied to watershed segmentation.

\section{Proposed Method}

\subsection{Extended fuzzy set}

Let $T, I$ and $F$ be real sub-set of $[0,1] . T$ is True, $I$ is Indeterminate, $F$ is False. $T, I$ and $F$ may be continuous or discrete. They are set-valued vector functions/operators depending on many parameters. They may overlap or converted from one to the other[2]. In our problem, when an image is transformed into fuzzy domain, three subsets $T, I$, and $F$ will be used to represent True, Indeterminate and False. A pixel can be represent as $\{t, i, f\}$, that means the pixel is $t \%$ true, $i \%$ indeterminacy and $f \%$ false. In classic Fuzzy Logic,

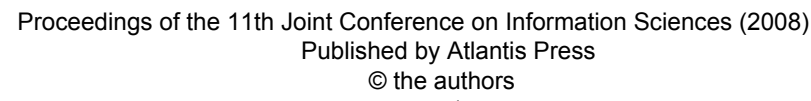


$i=0 ; 0 \leq t, f \leq 1 ; \bar{t}=f=1-t,$.

In Extended Fuzzy Logic, $\bar{t} \neq f$.

\subsection{Extended Fuzzy domain}

The goal of image segmentation is to extract object from image. Watershed segmentation method is good for nearly uniformed background and objects with blurred edges. We will take objects as $T$ and background as $F$. Because the blurred edges are gradually changed from objects to background; it is hard to separate from objects and background. The indeterminate values of edges are high.

Given an image, each pixel $P(i, j)$ in the image needs to be transformed to Extended Fuzzy domain:

$$
P_{E}(i, j)=\{T(i, j), I(i, j), F(i, j)\}
$$

. Where $T(i, j), I(i, j)$, and

$F(i, j)$ are defined as[3, 4]:

$T(i, j)=\frac{g(i, j)-g_{\min }}{g_{\max }-g_{\min }}$

$I(i, j)=S\left(g_{i j}, a, b, c\right)= \begin{cases}0 & 0 \leq g_{i j} \leq a \\ \frac{\left(g_{i j}-a\right)^{2}}{(b-a)(c-a)} & a \leq g_{i j} \leq b \\ 1-\frac{\left(g_{i j}-c\right)^{2}}{(c-b)(c-a)} & b \leq g_{i j} \leq c \\ 1 & g_{i j} \geq c\end{cases}$

$F(i, j)=1-T(i, j)$

Where $g_{i j}$ is the gray value of pixel $P(i, j) . a, b$ and $c$ can be determined by using method in[5].

Indeterminate domain is enhanced for further processing after the image project to Extended Fuzzy domain[6]: $I\left(\mu_{x}\left(g_{i j}\right)\right)=2 \mu_{x}^{2}\left(g_{i j}\right) \quad 0 \leq \mu_{x}\left(g_{i j}\right) \leq 0.5$ $I\left(\mu_{x}\left(g_{i j}\right)\right)=1-2\left(1-\mu_{x}\right)^{2} \quad 0.5 \leq \mu_{x}\left(g_{i j}\right) \leq 1$
2.3 Segmentation by using $I$

The value of $I(i, j)$ is used as the indeterminate degree of pixel $P(i, j)$. High $I$ value represents high uncertainty of a pixel.

In traditional Fuzzy Logic, $P(i, j)$ will belong to $T$ domain when the value of $T(i, j)$ is bigger than a threshold. In Extended Fuzzy domain we need consider both $T(i, j)$ and $I(i, j)$. If $I(i, j)$ is high, the indeterminate degree of $P(i, j)$ is high and it may not belong to $T$. The new definition of $T$ and $F$ on Extended Fuzzy domain consider $I$ inference.

$$
\begin{aligned}
& T_{E}(i, j)=T(i, j) I(i, j) \\
& F_{E}(i, j)=(1-F(i, j)) I(i, j)
\end{aligned}
$$

A threshold $t$ is calculated to determine $P(i, j)$ is in $T$ domain or $F$ domain.

$$
\begin{aligned}
& T(i, j)= \begin{cases}T & T_{E} \geq t \\
\bar{T} & T_{E}<t\end{cases} \\
& F(i, j)= \begin{cases}F & F_{E} \geq t \\
\bar{F} & F_{E}<t\end{cases}
\end{aligned}
$$

Because the edges are gradually change from objects to background, it is neither objects nor background.

$$
\text { Edge }=\bar{T} \cap \bar{F}
$$

The threshold $t$ and Edge can be calculated by the following heuristic method.

a. Let

$$
U(i, j)= \begin{cases}T(i, j) & g(i, j)<123 \\ F(i, j) & g(i, j) \geq 123\end{cases}
$$

b. Select an initial estimate threshold $t_{0}$. 
c. Separate $U$ by using $t_{0}$, and produces two groups of pixels: $G_{1}$ (domain $\bar{T}+\bar{F}$ ) and $G_{2}$ (domain $T+F$ ).

d. Calculate the average gray level values $\mu_{1}$ and $\mu_{2}$ for regions $G_{1}$ and $G_{2}$.

e. Compute the new threshold value:

$$
t=\frac{\mu_{1}+\mu_{2}}{2}
$$

f. Repeat steps 2 through 4 until the difference in $T$ is smaller than $\varepsilon$ in the two successive iterations. We choose $\varepsilon=0.001$ in our experiments.

g. Binary the image. For all the pixels with the $U(i, j)<t$, set 0 as their value. Set 1 for the rest of pixels.

\subsection{Watershed segmentation algorithm}

Because the watershed method is good for finding optimal segmentation, the following watershed method is applied to the binary image from 2.3 .

a. Find object or background region $R_{1}, R_{2}, \ldots, R_{m}$.

b. Use a $3 \times 3$ structure element to do dilation on these regions.

c. Build a dam at the place where two components get merged.

d. Repeat step c until all components merge together.

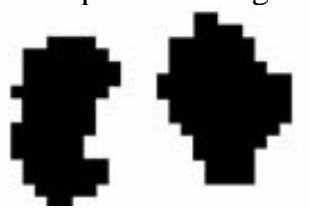

(a)

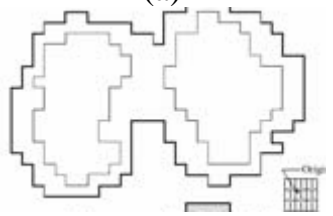

(b)

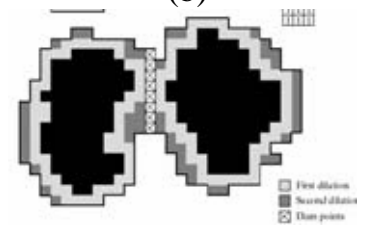

(c)

Figure 1: (a) Two object/background regions (b) Dilation of two components (c) Dam construction

\section{Experiment Results}

In figure 2 and 3, the Extended Fuzzy Logic method is compared with other segmentation algorithms: Sobel, Canny, and non-fuzzy watershed edge detector.

Figure 2(a) is a blurred cell image. The edge is ambiguous. (b) is the result by applying Sobel operator, the edges are not well detected. It is under-segmented (c) is using Canny operator, the edges are oversegmented. And the detection edges do not connect very well. (d) is using non-fuzzy watershed method in Matlab, the result show much oversegmentation. (e) is the result by using proposed method. The edges give good boundary for cells and they are smooth and connected.

The Extended Fuzzy Logic method is also noise tolerant. Figure 3(a) is coin image with Gaussian noise. The mean is 0 and the standard variance is 2.55 . The Sobel operator in (b) can remove most of noise, but the edges are over- 
segmented and the noise is not totally removed. The Canny operator in (c) and non-fuzzy watershed in (e) is noise sensitive. Both of them have over-segmented result. The extended fuzzy watershed method in (f) gives a clear and connected boundary.

The extended fuzzy watershed method also can be used on clear boundary image. See figure 4(a), it is a tablet image. The tablets are clear different with background. (b) is using non-fuzzy watershed method. The result is oversegmented. (c) is result by using the proposed method, it gets a good segmentation.

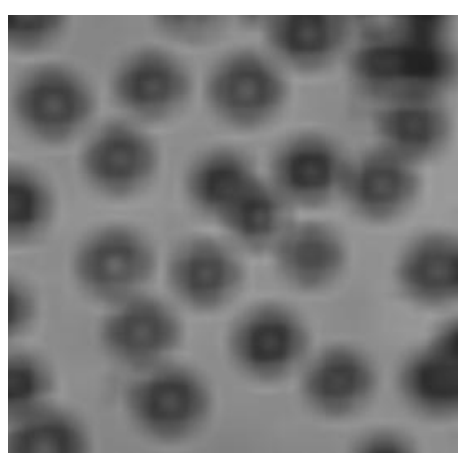

(a)

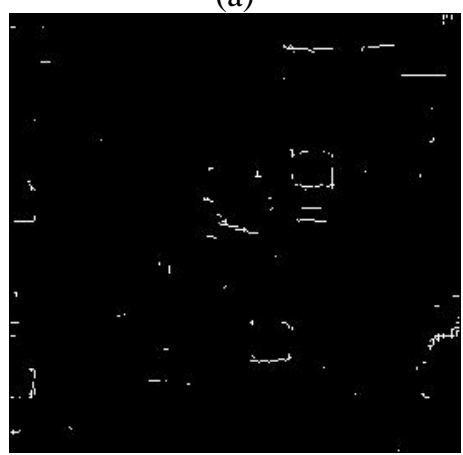

(b)

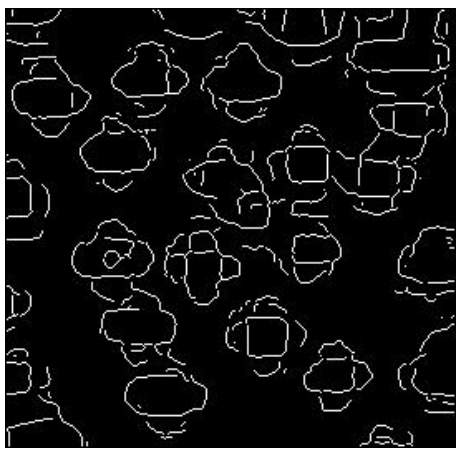

(c)

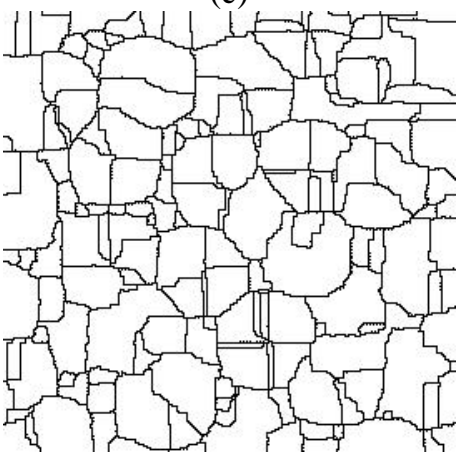

(d)

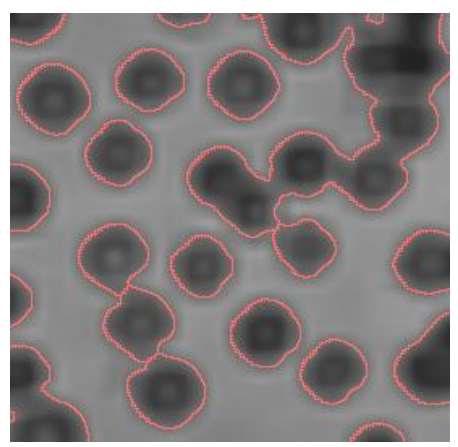

(e)

Figure 2: (a) The original cell image. (b) Sobel operator. (c) Canny operator. (d) Non-fuzzy watershed method. (e) Fuzzy watershed method number of bins is 10 


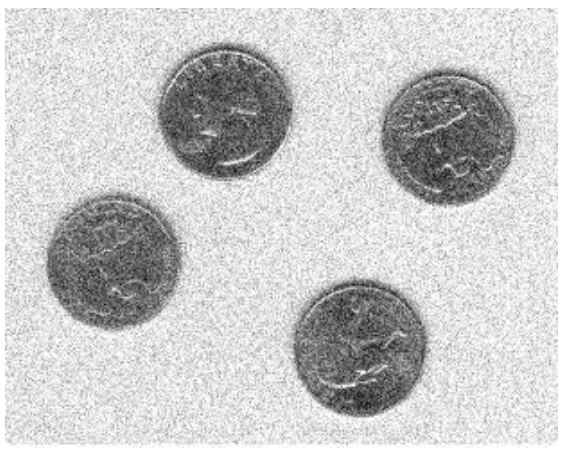

(a)

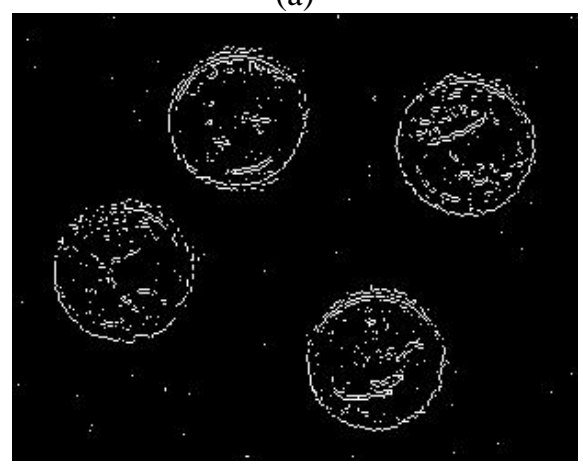

(b)

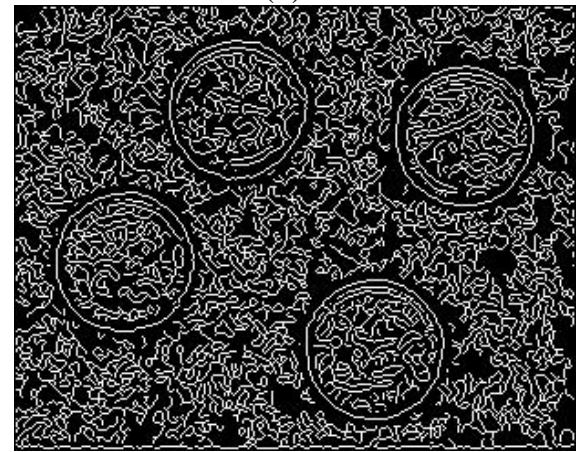

(c)

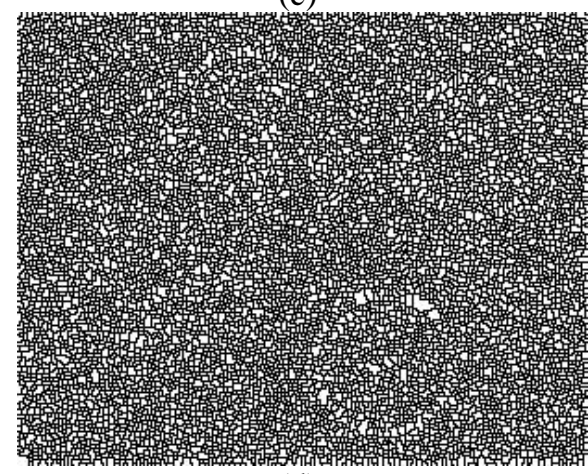

(d)

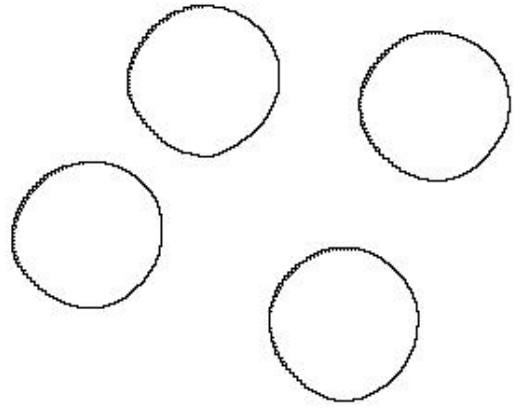

(e)

Figure 3: (a) The original pears image. (b) Sobel operator. (c) Canny operator. (d) Non-fuzzy watershed method. (e) Fuzzy watershed method, number of bins is 20

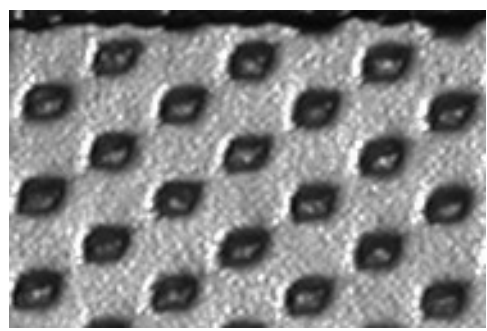

(a)

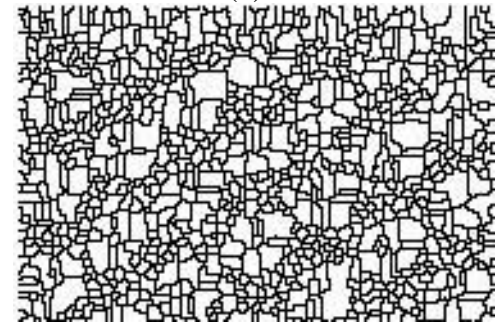

(b)

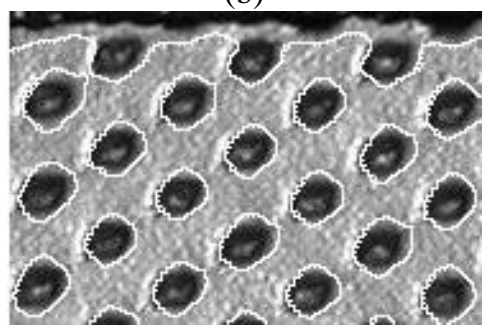

(c)

Figure 4: (a) Tablet image. (b) Watershed operator. (c) Extended Fuzzy Logic operator 


\section{Conclusion}

Fuzzy logic is successfully used in many fields. But there are a lot of problems cannot be solved by fuzzy logic. In this paper, we extended fuzzy logic into 3 domains: True, False and indeterminate. Because there are existing unknown conditions and factors in many problems. And the indeterminate domain is the important part in some problems. We apply the extended fuzzy logic in watershed segmentation problems. The results show that it much better than nonfuzzy method on solving blurred boundary images and it is also workable on clear boundary image. Another advantage of using extended fuzzy logic is noise tolerant than the original watershed method.
[1] L.A.Zadeh, "Fuzzy sets," Information and Control, vol. 8(3), pp. 383-353, 1965. [2] F. Smarandache, A Unifying Field in Logics Neutrosophic Logic. Neutrosophy, Neutrosophic Set, Neutrosophic Probability 3rd ed: American Research press, 2003.

[3] H. D. Cheng and J. G. Li, "Fuzzy Homogeneity and Scale Space Approach to Color Image Segmentation," Pattern Recognition, vol. 35, pp. 373-393, 2002. [4] Zadeh, e. al, and Eds, Fuzzy Sets and Their Application to Cognitive and

Decision Processes. London: Academic Press, 1975.

[5] H. D. Cheng, J. L. Wang, and X. J. Shi, "Microcalcification Detection using Fuzzy Logic and Scale Space Approaches," Pattern Recognition, vol. 37, pp. 363-375, 2004.

[6] R. C. Gonzalez and R. E. Woods, Digital Image Processing, 2 ed: Prentice Hall, 2002.

\section{Reference}

